Original article

\title{
Predicting opioid therapy safety in pancreatic cancer patients
}

\author{
Olga P. Bobrova ${ }^{1,2}$, Sergei K. Zyryanov ${ }^{3}$, Natalya A. Shnayder ${ }^{1,4}$, Marina M. Petrova ${ }^{1}$ \\ ${ }^{1}$ V.F. Voyno-Yasenetsky Krasnoyarsk State Medical University, Krasnoyarsk, Russia \\ ${ }^{2}$ Krasnoyarsk Regional Clinical Oncology Center, Krasnoyarsk, Russia \\ ${ }^{3}$ Peoples' Friendship University of Russia, Moscow, Russia \\ ${ }^{4}$ V.M. Bekhterev National Research Medical Center for Psychiatry and Neurology, St. Petersburg, Russia
}

Received 20 September 2020, Revised 12 October 2020, Accepted 24 October 2020

(C) 2020, Bobrova O.P., Zyryanov S.K., Shnayder N.A., Petrova M.M.

(C) 2020, Russian Open Medical Journal

Abstract: Background - Obligatory use of strong opioids for treating chronic pain syndrome in patients with pancreatic cancer provides the implementation of opioid-associated adverse reactions. Genetic and non-genetic risk factors are predictive of the opioid therapy safety. Contemporary methods of information analysis allow using prognostic risk models for practical application.

Objective - Identification of significant risk factors for the development of opioid-associated adverse drug reactions in patients with chronic pain syndrome against the background of pancreatic cancer.

Material and Methods - The study included 90 patients with chronic pain against the background of pancreatic cancer, randomized at a ratio of 1: 1 . Group 1 received morphine sulfate (MS), group 2 received fentanyl transdermal therapeutic system (FTTS) with standard adjuvant therapy (ketoprofen, diazepam, amitriptyline). To assess pain level, the 10-point Digital Rating Scale, the Visual Analogue Scale and the pain questionnaires were used. The assessment of the treatment safety was conducted by the Naranjo Scale. Assessment of prognostic genetic and non-genetic factors was carried out using ROC analysis with calculation of AUC (the area under the ROC-curve).

Results - Prognostic models of good quality were determined with the optimal ratio of sensitivity and specificity for the influence of genetic and non-genetic risk factors on the development of opioid-associated adverse drug reactions (OA-ADRs) in comparison groups. Various prognostic factors, complementing each other, were identified in the comparison groups.

Conclusion - The following OA-ADRs predicting factors were identified: for FTTS-associated nausea and vomiting - age and carriage of rs7438135 AG genotype of UGT2B7 gene; for local reactions - the sum of points on the ESAS scale and carriage of rs7438135 AA genotype of UGT2B7 gene; for difficulty urinating - the level of glomerular filtration rate; for neurotoxicity - the level of AST and bilirubin, and the carriage of rs1128503 GG genotype of $A B C B 1$ gene; for pruritus - carriage of rs 1045642642 AA genotype of $A B C B 1$ gene. The prognostic factors for the implementation of MS-associated neurotoxicity were age and comorbidity; for dry mouth was predicted best from the sum of points on the MMCE scale; weakness was predicted by the carriage of rs7668258 TT genotype of UGT2B7 gene.

Keywords: chronic pain syndrome, oncology, morphine sulfate, fentanyl transdermal therapeutic system, safety, adverse drug reactions, risk factors, personalized medicine, pharmacogenetics.

Cite as Bobrova OP, Zyryanov SK, Shnayder NA, Petrova MM. Predicting opioid therapy safety in pancreatic cancer patients. Russian Open Medical Journal 2020; 9: e0417.

Correspondence to Olga P. Bobrova. Address: Krasnoyarsk Regional Clinical Oncology Center, 16 Pervaya Smolenskaya St., Krasnoyarsk 660133, Russia. Phone: +79509933108. E-mail: bop 351971@mail.ru.

\section{Introduction}

High prevalence of chronic pain syndrome (CPS) in patients with pancreatic cancer determines the need for effective and safe analgesic therapy [1]. A feature of pain management in patients with pancreatic cancer is the obligatory use of strong opioids in combination therapy [2]. High-dose opioids, duration of use in patients with pancreatic cancer determines the implementation of opioid-associated adverse drug reactions (OA-ADRs) [3-4]. The severity of OA-ADRs varies in human population [5]. One of the urgent problems of practical oncopharmacology is the identification of prognostic factors for the safety of analgesic therapy [6-7].
It is known that genetic and non-genetic factors can influence the safety profile of opioid analgesics [8-10]. The palliative nature of patients with pancreatic cancer can also predetermine the change in safety profiles of analgesics due to multiple medicinal drug interactions [11-12]. Insufficient knowledge of the cumulative effect of genetic and non-genetic factors on safety of opioid therapy in patients with pancreatic cancer predetermined the urgency of our research.

Our study objective was to identify significant risk factors in the development of OA-ADRs in patients with CPS associated with pancreatic cancer. 
Table 1. Comparative characteristics of patients with pancreatic cancer $(n=90)$

\begin{tabular}{|c|c|c|c|}
\hline Indicators & Group $1(n=45) M S$ & Group $2(n=45)$ FTTS & $p$-level \\
\hline Age, years, Me (LQ, UQ) & $63(56-69)$ & $64.5(57-68)$ & $p^{1}=0.803$ \\
\hline Men, n (\%) & $21(43.75 \%)$ & $24(56.25 \%)$ & $p^{3}=0.337$ \\
\hline Women, n (\%) & $24(56.25 \%)$ & $21(43.75 \%)$ & $p^{3}=0.337$ \\
\hline $\mathrm{BMI}, \mathrm{kg} / \mathrm{m}^{2}, \mathrm{Me}(\mathrm{LQ}, \mathrm{UQ})$ & $21.67(19.84-24.38)$ & $21.9(21-22.7)$ & $p^{1}=1.000$ \\
\hline ECOG status, points, $\mathrm{M} \pm \mathrm{SD}$ & $1.67 \pm 0.48$ & $1.64 \pm 0.48$ & $\mathrm{p}^{2}=0.827$ \\
\hline Intensity of CPS according to DRS, points, Me (LQ, UQ) & $6(6-8)$ & $8(8-9)$ & $\mathrm{p}^{1}=0.060$ \\
\hline GFR CKD-EPI, ml/min, Me (LQ, UQ) & $84(75-98)$ & $83(69-97)$ & $p^{1}=0.849$ \\
\hline AST, units, Me (LQ, UQ) & $25(18-34)$ & $33(17.5-40)$ & $p^{1}=0.825$ \\
\hline ALT, units, Me (LQ, UQ) & $32(18.3-36)$ & $34.9(20.5-51.5)$ & $\mathrm{p}^{1}=0.079$ \\
\hline Bilirubin, $\mathrm{mmol} / \mathrm{I}, \mathrm{Me}(\mathrm{LQ}, \mathrm{UQ})$ & 19.1(10.7-58.7) & $23(13-34)$ & $p^{1}=0.067$ \\
\hline $\mathrm{T} 3, \mathrm{n}(\%)$ & $27(60 \%)$ & $29(64.44 \%)$ & $p^{3}=0.270$ \\
\hline $\mathrm{T} 4, \mathrm{n}(\%)$ & $18(40 \%)$ & $16(35.56 \%)$ & $p^{3}=0.833$ \\
\hline Total protein, g/l, Me (LQ, UQ) & $64(56-72)$ & $71(64-74.9)$ & $p^{1}=0.163$ \\
\hline Blood amylase, units per liter, $\mathrm{Me}(\mathrm{LQ}, \mathrm{UQ})$ & $42(34-54)$ & $40(32-52)$ & $p^{1}=0.372$ \\
\hline Hemoglobin, g/l, Me (LQ, UQ) & $121(107-127)$ & $123(110-132)$ & $\mathrm{p}^{1}=0.547$ \\
\hline Cancer-related weakness syndrome, $\mathrm{n}(\%)$ & $9(4.05 \%)$ & $13(5.85 \%)$ & $p^{3}=0.231$ \\
\hline Nutritional deficiency, $\mathrm{n}(\%)$ & $17(37 \%)$ & $12(26.67 \%)$ & $p^{3}=0.260$ \\
\hline Jaundice, $\mathrm{n}(\%)$ & $14(6.3 \%)$ & $16(7.2 \%)$ & $p^{3}=0.412$ \\
\hline Ascites, $\mathrm{n}(\%)$ & $5(2.25 \%)$ & $7(3.15 \%)$ & $p^{3}=0.379$ \\
\hline MMSE, points, $M \pm S D$ & $27 \pm 1.13$ & $26.04 \pm 2.15$ & $p^{2}=0.071$ \\
\hline Dyspeptic manifestations, $\mathrm{n}(\%)$ & $25(11.25 \%)$ & $29(13.05 \%)$ & $p^{3}=0.259$ \\
\hline ESAS, points, Me (LQ, UQ) & $2.62(2-3)$ & $2(2-3)$ & $\mathrm{p}^{1}=1.000$ \\
\hline Charlson comorbidity index, points, $M \pm S D$ & $5.00 \pm 1.71$ & $4.57 \pm 1.39$ & $p^{2}=0.455$ \\
\hline
\end{tabular}

AST, aspartate aminotransferase; ALT, alanine aminotransferase; BMI, body mass index; MS, morphine sulfate; GFR CKD-EPI, glomerular filtration rate according to the CKD-EPI (Chronic Kidney Disease Epidemiology Collaboration) scale; T3 and T4, stages of malignant neoplasia according to the international classification of tumors, revision 7; CPS, chronic pain syndrome; DRS, digital rating scale; FTTS, fentanyl transdermal therapeutic system; MMSE, MiniMental State Examination (scale for assessing mental status); ECOG (Eastern Cooperative Oncology Group), physical status assessment scale; ESAS, the Edmonton Symptom Assessment System. $\mathrm{p}^{1}-$ the Mann-Whitney comparison of means test, $\mathrm{p}^{2}-$ the Student's t-test for independent samples, $\mathrm{p}^{3}-$ the significance level of relative indicators $\left(x^{2}\right)$. Me (LQ, UQ), median with lower and upper quartiles; $M \pm S D$, mean with standard deviation; $n(\%)-$ frequencies in absolute values and in percentage.

Table 2. Structure and frequency of adverse drug reactions in patients with pancreatic cancer in comparison groups receiving opioid therapy (n=90)

\begin{tabular}{|c|c|c|c|c|}
\hline Adverse drug reaction & $M S(n=45)$ & FTTS $(n=45)$ & $x^{2}$ & p-level \\
\hline \multicolumn{5}{|c|}{ Gastrotoxicity } \\
\hline Constipation, n (\%) & $19(42.22 \%)$ & $5(11.11 \%)$ & 16.33 & $<0.001$ \\
\hline Nausea, vomiting, n (\%) & & $4(8.89 \%)$ & 8 & 0.005 \\
\hline Dry mouth, $\mathrm{n}(\%)$ & $5(11.11 \%)$ & & 10 & 0.002 \\
\hline \multicolumn{5}{|c|}{ Neurotoxicity } \\
\hline Sedation (sleepiness), n (\%) & $5(11.11 \%)$ & 10 (22.22\%) & 3.33 & 0.068 \\
\hline Weakness (adynamia), n (\%) & $5(11.11 \%)$ & $5(11.11 \%)$ & 0.00 & 1.000 \\
\hline Dizziness, $\mathrm{n}(\%)$ & & $5(11.11 \%)$ & 10 & 0.002 \\
\hline Disorientation, $\mathrm{n}(\%)$ & & $2(4.44 \%)$ & 4.0 & 0.046 \\
\hline \multicolumn{5}{|c|}{ General and local reactions } \\
\hline Itchy skin, n (\%) & $7(15.56 \%)$ & $7(15.56 \%)$ & 0.00 & 1.000 \\
\hline Local reactions in the area of application, $n(\%)$ & & $3(6.66 \%)$ & 6.0 & 0.143 \\
\hline \multicolumn{5}{|c|}{ Ürotoxicity } \\
\hline Difficulty urinating, $n, \%$ & $3(6.66 \%)$ & $2(4.44 \%)$ & 0.40 & 0.527 \\
\hline
\end{tabular}

MS, morphine sulfate; FTTS, fentanyl transdermal therapeutic system. $\mathrm{n}(\%)$ - frequencies in absolute values and in percentage.

Table 3. Severity of adverse drug reactions in comparison groups: patients with pancreatic cancer $(n=90)$

\begin{tabular}{lcccr}
\hline Severity of ADRs & Number of adverse reactions & $X^{2}$ & p-level \\
& MS $(\mathrm{n}=45)$ & FTTS $(\mathrm{n}=45)$ & & \\
\hline Mild degree & $0 \%$ & $0 \%$ & & \\
Average degree & $22 \%$ & $28 \%$ & 1.44 & 0.230 \\
Severe degree & $22 \%$ & $12 \%$ & 3.48 & 0.062 \\
\hline ADRs, adverse drug reactions; & MS, morphine sulfate; & FTTS, fentanyl \\
transdermal therapeutic system. & & &
\end{tabular}

\section{Material and Methods}

\section{Characteristics of patients with pancreatic cancer}

Using random sampling, according to the $\mathrm{MCB} X$ revision code C25 (1995), 90 patients with CPS, against the background of pancreatic cancer, at the age of $18-75$ years old were included into the study. The patients were randomized by medication they took and intensity of CPS, taking into account clinical guidelines (group 1: $n=45$, morphine sulfate (MS); group 2: $n=45$, fentanyl transdermal therapeutic system - FTTS). Inclusion criteria were: verified pancreatic cancer, fewer than 3 points on the physical status assessment scale sensu the Eastern Cooperative Oncology Group (ECOG), ongoing combined treatment, opioid-naive patients. The study did not include patients with a glomerular filtration rate (GFR) of less than $15 \mathrm{ml} / \mathrm{min}$, high activity of hepatic aminotransferases (> 3 norms), respiratory failure (above stage 3), hypersensitivity to opioids, cachexia, epilepsy, and concomitant administration or a period of up to 14 days from the moment of stopping taking monoamine oxidase inhibitors (MAO). The observation period was $5.95 \pm 0.67$ months in the group receiving morphine sulfate and $5.73 \pm 0.84$ months in the group receiving fentanyl TTS.

The patients of the compared groups, included in the study, were comparable in terms of age and sex characteristics, anthropometric indicators, laboratory characteristics, the results of histological verification, comorbidity, the volume of combined treatment, and the structure of pharmacotherapy for concomitant pathology. Clinical characteristics of the patients are presented in Table 1. 


\section{Study design}

According to the study protocol, patients in the compared groups received strong opioid analgesics against the background of standard adjuvant therapy (ketoprofen, diazepam, amitriptyline), taking into account the pathogenetic features of CPS in patients with pancreatic cancer

Table 4. ROC analysis indices of non-genetic risk factors for developing OA-ADRs in patients with CPS associated with pancreatic cancer (in the morphine sulfate group, $n=45$ )

\begin{tabular}{|c|c|c|c|c|c|c|}
\hline \multirow[b]{2}{*}{$O A-A D R s$} & \multicolumn{5}{|c|}{ Confidence interval } & \multirow[b]{2}{*}{$p$} \\
\hline & AUC Cutpoint & $\begin{array}{l}\text { Lower } \\
\text { bound }\end{array}$ & $\begin{array}{l}\text { Upper } \\
\text { bound }\end{array}$ & Sen, $\%$ & Sp, \% & \\
\hline & \multicolumn{6}{|c|}{ Patient age } \\
\hline \multirow{3}{*}{ Sedation } & $0.741 \geq 64.5$ & 0.584 & 0.898 & 80 & 64 & 0.082 \\
\hline & & & AMSE & & & \\
\hline & $0.723 \geq 26.5$ & 0.474 & 0.971 & 80 & 40 & 0.108 \\
\hline \multirow{3}{*}{ Weakness } & & & ĀST & & & \\
\hline & $0.728 \geq 31.5$ & 0.519 & 0.936 & 80 & 63 & 0.100 \\
\hline & $0.785 \geq 5.5$ & 0.644 & 0.925 & 80 & 70 & 0.040 \\
\hline \multirow{4}{*}{ Dry mouth } & & & AMCE & & & \\
\hline & $0.790 \geq 25.5$ & 0.553 & 1.000 & 80 & 77 & 0.037 \\
\hline & & Slomeru & Filtrati & Rate & & \\
\hline & $0.697 \leq 85.5$ & 0.508 & 0.887 & 100 & 44 & 0.155 \\
\hline & \multicolumn{6}{|c|}{ Operative treatment } \\
\hline Itchy skin & 0.705 & 0.513 & 0.897 & 86 & 55 & 0.088 \\
\hline \multirow{3}{*}{ Difficulty urinating } & \multicolumn{5}{|c|}{ Dyspepsia } & \\
\hline & 0.786 & 0.461 & 1.000 & 67 & 90 & 0.101 \\
\hline & $0.770 \leq 24.005$ & $\begin{array}{l}\text { Bod } \\
0.643\end{array}$ & $\begin{array}{l}\text { nass inc } \\
0.897\end{array}$ & 100 & 74 & 0.122 \\
\hline \multirow{2}{*}{ Pharmacoresistance } & & & protei & & & \\
\hline & $0.694 \leq 59.5$ & 0.63 & 0.524 & 77 & 60 & 0.864 \\
\hline
\end{tabular}

AST, aspartate aminotransferase; $\mathrm{Cl}$, confidence interval; $\mathrm{BMI}$, body mass index; GFR, glomerular filtration rate; AUC, area under the ROC-curve; MMSE, Mini-Mental State Examination; Sen, sensitivity; OA-ADRs, opioidassociated adverse drug reactions; Sp, specificity.

Table 5. ROC analysis indices of non-genetic risk factors for developing OA-ADRs depending on genetic factors in patients with CPS associated with pancreatic cancer (in the morphine sulfate group, $n=45$ )

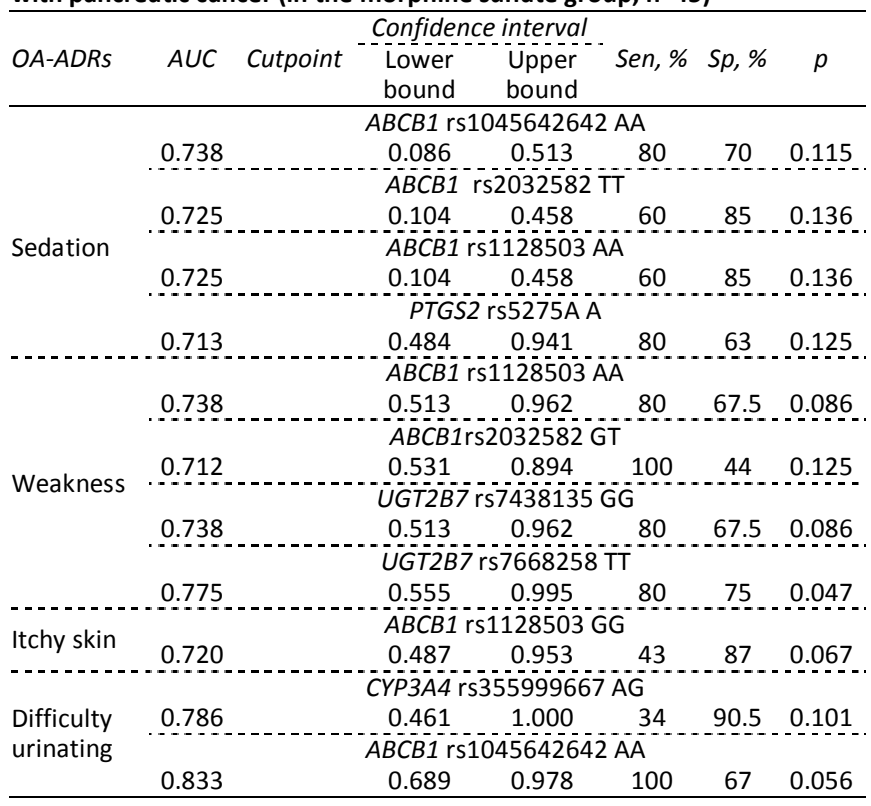

$\mathrm{Cl}$, confidence interval; $\mathrm{AUC}$, area under the ROC-curve; OA-ADRs, opioidassociated adverse drug reactions; Sen, sensitivity; Sp, specificity.
According to clinical indications, prosidol in transbuccal tablets and morphine hydrochloride in solution were used to relieve unbearable pain, depending on its intensity.

In patients with the neuropathic component of pain syndrome, diagnosed by the DN4 questionnaire, gabapentinoids were added to the dose titration therapy regimen.

The intensity of CPS was determined, using a 10-point digital rating scale (1-4/10 points - mild pain; $5 / 10$ points - moderate pain; $6-9 / 10$ points - severe pain; $10 / 10$ points - very severe pain) and 4 points of the Visual Analogue Scale ( $1 b-$ mild pain, $2 b-$ moderate pain, $3 b$ - severe pain, $4 b$ - very severe pain), with mandatory visual grading by the patient himself/herself. When assessing the intensity of CPS, the complex influence of the emotional component, gender and age characteristics, and general physical status were taken into account [13]. To objectify the assessment of pain, along with analgesic scales, self-modified pain questionnaires were used with visualization of possible characteristics, sites of localization and irradiation of pain syndrome.

The general condition of a cancer patient with pancreatic cancer was assessed according to the ECOG scale ( 0 - 4 points) [14]. The pancreatic cancer staging was carried out according to the international classification of stages of malignant neoplasms TNM, revision 7 [15]. Written consent was obtained from each study participant.

All patients underwent a standard clinical and laboratory examination. The functional state of the kidneys was assessed by the level of GFR calculated by the CKD-EPI (Chronic Kidney Disease Epidemiology Collaboration) scale [16].

The scope of instrumental studies included esophagogastroduodenoscopy, multi-slice computed tomography (MSCT) of the chest, ultrasound of abdominal organs, and magnetic resonance imaging (MRI) of the abdominal organs [17].

All patients were examined by a general practitioner and/or endocrinologist to correct the existing comorbid pathology of a competitive or concomitant nature.

\section{Methods for assessing the effectiveness and safety of opioid therapy}

Evaluation of the therapy effectiveness was carried out for patients in the compared groups, according to the CRS and life quality indicators on the Edmonton Symptom Assessment System (ESAS) scale. The latter is the scale for assessing symptoms in palliative medicine. The significance of the relationship between adverse drug reactions (ADRs) and opioids in patients with pancreatic cancer was determined according to the Naranjo scale and algorithms of F.E. Karch and L. Lasagna. Cognitive status was assessed using the scale for assessing mental status: the MiniMental State Examination (MMSE).

\section{Molecular genetic methods}

Within the framework of molecular genetic research, the study of single nucleotide variants (SNVs) of genes was carried out: $A B C B 1$ (rs1045642, rs2032582, rs1128503); OPRM1 (rs 1799971); UGT2B7 (rs 7668258, rs12233719, rs7438135); CYP3A4 (rs2740574, rs35599367); CYP3A5 (rs776746); IL1B (rs1143627); PTGS2 (rs5275); LOC541472 (rs1800795) by real-time polymerase chain reaction (PCR-RT) on the Rotor-Gene 6000 real time DNA amplification system (Corbett Life Science, Australia), using 
TaqMan allelic discrimination technology and commercially available fluorescent probes (Applied Biosystems, USA; Syntol, RF).

\section{Statistical analysis}

Statistical data processing was performed using the IBM SPSS ${ }^{\circledR}$ Statistics 20.0 software package (USA). The normal distribution was tested using the Kolmogorov-Smirnov and the Shapiro-Wilk tests. Descriptive statistics for nonparametric data were presented in the form of a median, and the low and the upper quartiles - Me (LQ, $\mathrm{UQ}$ ); for parametric data, we used arithmetic mean and standard deviation - M $\pm S D$. The significance of differences in categorical features was assessed using the Chi-Square Test of Independence. The Student's t-test for parametric data and the Wilcoxon signedrank test for nonparametric data were used to assess the statistical significance of differences among two samples. Differences were considered significant at $\mathrm{p}<0.05$. ROC analysis (receiver operating characteristics) with the calculation of AUC (area under the ROCcurve), cutpoints with sensitivity and specificity, likelihood ratio (LR) and predictive value (PV) were used to create a prediction scale.

\section{Results}

The median of a single dose of MS during treatment was 120 (90-180) $\mathrm{mg}$ and increased by $56 \%$ during the study period. The dose of FTTS was $100(75,150) \mathrm{mcg}$ and increased by $32 \%$ during the study period. The increase in the dose of opioid analgesics over 6 months of therapy can be explained by development of tolerance and progression of the disease.

Table 6. Indicators of the model for calculating the risks of developing OA-ADRs depending on non-genetic factors in patients with CPS associated with pancreatic cancer (in the fentanyl TTS group, $n=45$ )

\begin{tabular}{|c|c|c|c|c|c|c|c|}
\hline \multirow[b]{2}{*}{$O A-A D R s$} & \multicolumn{7}{|c|}{ Confidence interval } \\
\hline & $A \cup C$ & Cutpoint & $\begin{array}{l}\text { Lower } \\
\text { bound }\end{array}$ & $\begin{array}{l}\text { Upper } \\
\text { bound }\end{array}$ & Sen, $\%$ & Sp, \% & $p$ \\
\hline \multirow{4}{*}{$\begin{array}{l}\text { Nausea, } \\
\text { vomiting }\end{array}$} & \multicolumn{7}{|c|}{ Patient age } \\
\hline & 0.78 & 67.5 & 0.56 & 1 & 75 & 76 & 0.067 \\
\hline & \multicolumn{7}{|c|}{ BMI } \\
\hline & 0.765 & 27.25 & 0.521 & 1 & 75 & 78 & 0.125 \\
\hline \multicolumn{8}{|c|}{ Comorbidity } \\
\hline \multirow{4}{*}{ Local reaction } & 0.821 & 5.5 & 0.645 & 0.998 & 67 & 79 & 0.065 \\
\hline & \multicolumn{7}{|c|}{ ESAS score } \\
\hline & 0.881 & 3.5. & 0.783 & 0.979 & 100 & 83 & 0.029 \\
\hline & \multicolumn{7}{|c|}{ Total protein } \\
\hline \multirow{3}{*}{$\begin{array}{l}\text { Difficulty } \\
\text { urinating }\end{array}$} & 0.837 & 72.5 & 0.675 & 1. & 100 & 72 & 0.110 \\
\hline & \multicolumn{7}{|c|}{ GFR } \\
\hline & 0.737 & 74 & 0.543 & 0.931 & 71 & 53 & 0.049 \\
\hline \multirow{8}{*}{ Disorientation } & \multicolumn{7}{|c|}{ AST } \\
\hline & 0.872 & 38.5 & 0.677 & 1 & 100 & 73 & 0.078 \\
\hline & & & ESA & core & & & \\
\hline & 0.762 & 2.5 & 0.566 & 0.957 & 100 & 53 & 0.215 \\
\hline & & & Com & bidity & & & \\
\hline & 0.628 & 4.5 & 0.462 & 0.794 & 100 & 51 & 0.545 \\
\hline & \multicolumn{7}{|c|}{ GFR } \\
\hline & 0.895 & 92 & 0.757 & 1.000 & 100 & 79 & 0.061 \\
\hline \multirow{4}{*}{ Sedation } & \multicolumn{7}{|c|}{ AST } \\
\hline & 0.717 & 35.5 & 0.532 & 0.901 & 60 & 70 & 0.038 \\
\hline & \multicolumn{7}{|c|}{ Total bilirubin } \\
\hline & 0.818 & 21.5 & 0.667 & 0.969 & 70 & 30 & 0.002 \\
\hline
\end{tabular}

AST, aspartate aminotransferase; $\mathrm{Cl}$, confidence interval; $\mathrm{BMI}$, body mass index; GFR, glomerular filtration rate; AUC, area under the ROC-curve; ESAS, Edmonton Symptom Assessment System; OA-ADRs, opioidassociated adverse drug reactions; Sen, sensitivity; Sp, specificity.

\section{Characterization of opioid-associated adverse drug reactions}

The incidence of OA-ADRs in the compared groups was $97.77 \%$ (MS group) and $91.1 \%$ (FTTS group) with no statistical significance $(p=0.415)$. As seen in the Table 2 , the compared groups of patients differed statistically in a significant way in the frequency of developing constipation $(p<0.001)$, nausea and vomiting $(p=0.005)$, dry mouth and dizziness ( $p=0.002)$, and disorientation ( $p=0.046)$.

Thus, use of MS versus FTTS was characterized by a statistically significant prevalence of gastrointestinal OA-ADRs versus neurotoxic OA-ADRs. There were no statistically significant differences in the frequencies of occurrence of general and local cutaneous OA-ADRs and the development of difficulty urinating in the compared groups. The patients in the compared groups did not have OA-ADRs of mild severity. In terms of severity of OAADRs, the patients in the study groups did not have statistically significant differences (Table 3). Thus, according to the clinical data, both MS and FTTS had similar safety profiles.

\section{Predictive factors for implementation of adverse drug reactions}

The method of binary logistic regression was used to determine the cumulative contribution of the factors under study to the development of specific OA-ADRs. The following factors were considered predictors of the onset of the OA-ADRs. Clinical factors included gender, age, localization of pancreatic cancer, pathogenetic variant of CPS, type of surgical treatment, physical status sensu the physical status assessment scale (ECOG 0/6), presence of jaundice, cancer-related weakness, comorbidity, dyspepsia, ascites, body mass index (BMI) $0 / 6$, mental status on the MMSE scale $0 / 6$, and quality of life indicators on the ESAS scale $0 / 6(0-$ at the time of inclusion into the study, 6 - after six months of therapy). Laboratory factors included GFR $0 / 6$, aspartate aminotransferase (AST) $0 / 6$, alanine aminotransferase (ALT) $0 / 6$, bilirubin $0 / 6$, total protein $0 / 6$, hemoglobin $0 / 6$, leukocytes $0 / 6$, lymphocytes $0 / 6$, platelets $0 / 6$, erythrocytes $0 / 6$, glucose $0 / 6$, and amylase $0 / 6$. The following genetic factors were studied, including SNVs: rs1045642, rs2032582 and rs1128503 of $A B C B 1$ gene; rs1799971 of OPRM1 gene; rs7668258, rs12233719 and rs7438135 of UGT2B7 gene; rs2740574 and rs35599367 of CYP3A4 gene; rs776746 of CYP3A5 gene; rs1143627 of IL1B gene; rs5275 PTGS2; and rs1800795 of LOC541472 gene.

Prognostic significance of non-genetic and genetic factors in development of morphine-associated adverse drug reactions

Predictive value of the studied factors was determined by the area under the ROC-curve (AUC) [18]. In the graphical analysis of ROC-curves (receiver operating characteristics curves), the principle of the curve proximity to the upper left corner of the coordinate grid was pursued. Quality of predictive models was assessed, according to the expert scale for the AUC values: 0.5-0.6 - unsatisfactory; 0.6-0.7 - average; 0.7-0.8 - good; 0.8-0.9 - very good; 0.9-1.0 - excellent.

Only those factors that demonstrated the best AUC were subjected to further analysis. In the models of developing OAADRs, various risk factors exhibited different ratios of specificity and sensitivity (Table 4). 
Table 7. Indicators of the model for calculating the risks of developing OA-ADRs depending on genetic factors in patients with CPS associated with pancreatic cancer (in FTTS group, $n=45$ )

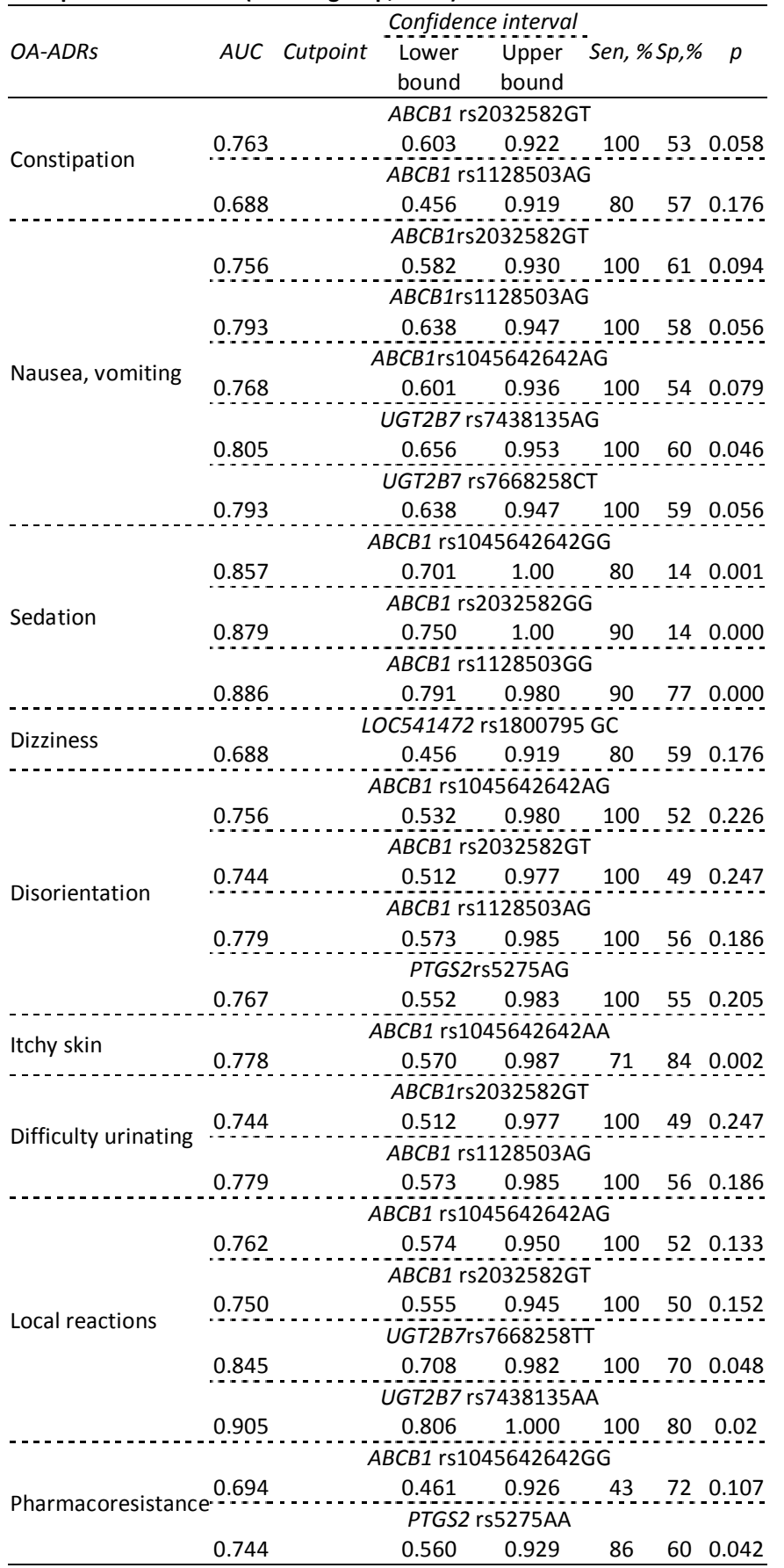

$\mathrm{Cl}$, confidence interval; AUC, area under the ROC-curve; OA-ADRs, opioidassociated adverse drug reactions; Sen, sensitivity; Sp, specificity.

The top quality models for determining prognostic non-genetic risk factors in developing OA-ADRs with statistical significance, in MS group, were: age $\geq 64.5$ years for the development of sedation; AST $\geq 31.5$ units and a comorbidity index of over 5.5 for the development of weakness; MMSE $\geq 25.5$ points for the development of dry mouth; BMI $\leq 24.005$ and presence of dyspepsia for the development of difficulty urinating.
These models showed the maximum agreement between sensitivity and specificity with a statistical significance level of under 0.05 and an AUC level of over 0.7. From the tabular analysis of genetic risk factors for OA-ADRs development, the optimal cutoff point was the sensitivity index $(80 \%)$ and the specificity index $(63 \%-85 \%)$. The range of the area under the curve indicated the average predictive quality of the model (Table 5).

Among the studied SNVs in the models of medium predicting quality with statistical significance were: rs1045642642 AA of $A B C B 1$ gene and rs5275 AA of PTGS2 gene - for the occurrence of sedation; rs1128503 AA of $A B C B 1$ gene, rs7438135 GG and rs7668258 TT of UGT2B7 gene - for the emergence of weakness. The specificity and sensitivity of the model were $67.5-75 \%$ and $80 \%$, respectively.

The ideal model should have $100 \%$ sensitivity and specificity. The boundary of the optimal close ratio of specificity and sensitivity of the predictive model was implied by the optimal cutoff value. The cutoff value facilitated practical application of the model. A good predictive model was reflected by the association of rs1045642642 AA of $A B C B 1$ gene with the development of difficulty urinating in the MS group.

Prognostic significance of non-genetic and genetic factors in development of fentanyl-associated adverse drug reactions

The analysis of modeling prognostic non-genetic factors for implementation of OA-ADRs in the FTTS group demonstrated the construction of models of very good quality with $100 \%$ specificity and $83 \%$ sensitivity with a cutoff limit of above 3.5 points on the ESAS scale for the development of local ADRs; with $100 \%$ specificity and $79 \%$ sensitivity with a cutoff limit of over $92 \mathrm{ml} / \mathrm{min}$ of GFR level for the development of disorientation; and with $60 \%$ specificity and $70 \%$ sensitivity with a cutoff limit of over 35.5 units of AST level for the development of disorientation.

Good quality model building with $75 \%$ specificity and $76 \%$ sensitivity with a cutoff over 67.5 years by age and with $75 \%$ specificity and $78 \%$ sensitivity with a cutoff of over $27.25 \mathrm{BMI}$ was shown for development of nausea and vomiting.

The analysis of non-genetic factors demonstrated the construction of models for predicting OA-ADRs in the FTTS group with statistical significance (Table 6).

A model for constructing a very good quality genetic risks of developing OA-ADRs in the FTTS group was shown in carriers rs1128503 GG of $A B C B 1$ gene for statistically significant development of sedation with $90 \%$ sensitivity and $77 \%$ specificity.

With $100 \%$ sensitivity and $67 \%$ specificity, a statistically significant predictive model of nausea and vomiting was demonstrated for rs7438135AG of UGT2B7 gene. With $100 \%$ sensitivity and $70 \%$ specificity, a statistically significant model for predicting the development of local reactions was shown for rs 7668258 TT of UGT2B7 gene, and with $100 \%$ sensitivity and $72 \%$ specificity for rs7438135 AA of UGT2B7 gene.

The constructed models of average quality demonstrated a statistically significant prognostic value for development of pharmacoresistance in carriers of rs5275 AA of PTGS2 gene with $86 \%$ sensitivity and $60 \%$ specificity; and for the development of pruritus rs $1045642642 \mathrm{AA}$ of $A B C B 1$ gene with $86 \%$ sensitivity and $60 \%$ specificity (Table 7). 


\section{Discussion}

In this study, individual criteria for the OA-ADRs occurrence were considered and compared with each other. The analysis of the obtained indicators of the models for predicting OA-ADRs risks factors in the comparison groups exhibited different statistically significant diagnostic values of the likelihood of occurrence of OAADRs with different specificity, sensitivity and model quality. The results of the repeated clarifying ROC analysis with the exclusion of insignificant factors from the analysis demonstrated the values of the area under the ROC-curve $\geq 0.7$ in nearly all obtained models in the compared groups. The demonstrated good quality of the ROC-curve was optimal for the practical use of the models [18].

However, only some models showed the presence of an important condition of practical likelihood which is the correspondence between the levels of sensitivity and specificity. The obtained cutoff points (criteria) in models of good and very good quality allow them to be used in practice. According to the results of our study, no genetic and non-genetic risk factors were found for the occurrence of constipation, taking into account the low quality of the constructed prognostic models. The absence of predictive factors for opioid-associated constipation in patients with pancreatic cancer did not match the data of previous studies bt Laugsand E.A. et al. (2015). Performance status of the patients sensu Karnovsky, the presence of metastases, the type of laxative, physical activity, hospitalization, carriage of SNVs of TPH1, OPRM1, $A B C B 1, C H R M 3, C O M T$ genes were factors for development of constipation $(p \leq 0.001)$ in patients with oncological profile [19]. This fact can be explained by the structural differences of the studied genetic and non-genetic factors, heterogeneity of used opioids, variety of localizations of malignant neoplasms, compared with our study.

The limited sample size of patients with pancreatic cancer in our study may have also provided the specificity of the collected data only for this category of patients. Genotyping of 45 ONVs demonstrated the association of SNVS of AIM1L, CLCC1, MUC16, PDE3A, POM121L2 and ZNF165 genes with the risk of opioidassociated constipation [20]. In Italian cancer patients, the presence of an association of two SNVs in the ZNF568 and PDE3A genes with the risk of opioid-associated constipation was also shown [20]. The diversity of the studied genes, as well as ethnic and territorial characteristics, could explain the inconsistency of our results. However, each published study indicated the need for further research of possible genetic predictors of the OA-ADRs implementation [21]. The absence of predicting factors for the development of constipation even in the MS group in our study also requires further investigation of the complex influence of genetic and nongenetic risk factors.

It was not possible to predict non-genetic risk factors for the development of dizziness, sedation, weakness, pruritus in the FTTS group with high specificity and sensitivity. This finding is consistent with a few contradictory foreign studies [22]. In the MS group, certain risk factors for OA-ADRs development with high specificity and sensitivity (level of total protein in serum - for pharmacoresistance; comorbidity - for weakness; old age - for sedation) require further study to determine the contribution of each particular factor to the likelihood of developing these ADRs.

Prognostic genetic risk factors for the development of opioidassociated dizziness and disorientation, difficulty urinating in both compared groups have not been determined; same was true for pharmacoresistance and itchy skin in the MS group. The absence of an opioid dose in the structure of predicting factors for the development of FTTS-associated nausea and vomiting in our study corresponded to the previously obtained data on the absence of dose-dependence in the implementation of the above-mentioned ADRs [22].

Cumulative mutual influence of genetic and non-genetic factors should form the basis for further research. Certain prognostic genetic markers for the implementation of sedation (rs1045642642 AA, rs2032582 TT, rs1128503 AA of ABCB1 gene, rs5275 AA of PTGS2 gene in the MS group) were not statistically significant in comparison with rs $1128503 \mathrm{GG}$ of $A B C B 1$ gene in the FTTS group ( $p=0.000$ ), weakness in the MS group ( $r$ 7668258 $\pi$ of UGT2B7 gene, $p=0.047$ ), pruritus in the FTTS group ( $r s 1045642642$ $A A$ of $A B C B 1$ gene, $p=0.002$ ), and pharmacoresistance in the FTTS group (rs5275AA of PTGS2 gene, $\mathrm{p}=0.042$ ). This fact should be taken into account when conducting analgesic therapy with strong opioids in the patients with pancreatic cancer.

In our study, no cognitive impairment was recorded according to the MMSE screening scale, and no significant predictors of prognosis were obtained. This fact can be explained by the shortterm study, the use of dose-saving opioid therapy of MS and FTTS as part of a combination treatment, as well as the only studied nosology - pancreatic cancer. Currently, significant risk factors for opioid-associated cognitive dysfunction are known: advanced age, low Karnovsky status, absence of unbearable pain, lung cancer, daily dose of MS over $400 \mathrm{mg}$ or an equivalent dose of another opioid, less than 15 months from the date of diagnosis [23]. The obtained results could be highly specific solely for the studied sample, because of its small size. In course of this study, various prognostic factors were identified in the comparison groups.

A variety of predictive factors for the implementation of OAADRs in comparison groups could complement each other when conducting associative analysis. The obtained levels of AUC for ROC-curves predetermine further construction of the logistic regression equation. The interrelations of predictive risk factors for development of undesirable reactions among those factors determine the multiplicative effect of interaction. Logistic regression makes it possible to rank the contribution of each individual prognostic criterion within the combined effect in an individual patient. Structural differences in predictive factors predetermine a study of cumulative effect of genetic and nongenetic factors on OA-ADRs development.

\section{Conclusion}

For the first time, the study of the association of non-genetic and genetic factors with the risk of developing OA-ADRs was carried out. The main non-genetic factors in predicting the development of MS-associated ADRs were: for developing neurotoxicity - age over 64.5 years and Charlson comorbidity index over 5.5; for implementation of dry mouth - less than 25.5 points on the MMSE scale. The group of non-genetic factors for the prognosis of FTTS-associated ADRs included: for development of nausea and vomiting - age over 67.5 years; for local ADRs over 3.5 points on the ESAS scale; for difficulty urinating - the level of GFR above $74 \mathrm{ml} / \mathrm{min}$; for developing neurotoxicity - AST level over 35.5 units and bilirubin level over $21.5 \mathrm{mmol} / \mathrm{l}$. Carriage of rs7438135 AG and rs7438135 AA of UGT2B7 gene, rs1128503 GG and rs1045642642 AA of $A B C B 1$ gene can be used as markers of FTTS-associated local skin ADRs, sedation, and itchy skin, 
correspondingly. Carriage of rs7668258 TT of UGT2B7 gene can be used as a genetic marker of MS-associated weakness.

\section{Ethical approval}

All procedures performed in studies, involving human participants, were in accordance with the ethical standards of the institutional and national research committee and with the 1964 Declaration of Helsinki and its later amendments or comparable ethical standards.

\section{Funding}

This study was carried out as part of implementing the Federal Assignment No. 056-00119-18-00 Development of personalized approaches to safe and effective treatment of chronic pain in patients with malignant neoplasms.

\section{Conflict of interest}

We declare that we have no conflict of interest.

\section{References}

1. Lahoud MJ, Kourie HR, Antoun J, El Osta L, Ghosn M. Road map for pain management in pancreatic cancer: A review. World J Gastrointest Oncol 2016; 8(8): 599-606. https://doi.org/10.4251/wjgo.v8.i8.599.

2. Dobosz $\measuredangle$, Kaczor M, Stefaniak TJ. Pain in pancreatic cancer: review of medical and surgical remedies. ANZ J Surg 2016; 86(10): 756-761. https://doi.org/10.1111/ans.13609.

3. Vargas-Schaffer $\mathrm{G}$. Is the WHO analgesic ladder still valid? Twenty-four years of experience. Can Fam Physician 2010; 56(6): 514-517, e202e205. https://pubmed.ncbi.nlm.nih.gov/20547511/.

4. Barratt DT, Klepstad P, Dale O, Kaasa S, Somogyi AA. Innate immune signalling genetics of pain, cognitive dysfunction and sickness symptoms in cancer pain patients treated with transdermal fentanyl. $\begin{array}{llll}\text { PLoS One 2015; 10(9): } 0137179 & \end{array}$ https://doi.org/10.1371/journal.pone.0137179.

5. Evans HC, Easthope SE. Transdermal buprenorphine. Drugs 2003; 63(19): 1999-2010. https://doi.org/10.2165/00003495-200363190$\underline{00003}$

6. Yang $Q$, Xie DR, Jiang ZM, Ma W, Zhang YD, Bi ZF, et al. Efficacy and adverse effects of transdermal fentanyl and sustained-release oral morphine in treating moderate-severe cancer pain in Chinese population: a systematic review and meta-analysis. J Exp Clin Cancer Res 2010; 29(1): 67. https://doi.org/10.1186/1756-9966-29-67.

7. Gazenkampf AA, Khinovker VV, Pelipeckaya EYu, Pozharitckaia DV. Organization of chronic pain syndrome treatment by the example of Spanish healthcare system. Siberian Medical Review 2019; (3): 16-23. Russian. https://doi.org/10.20333/2500136-2019-3-16-23.

8. Koulouris Al, Banim P, Hart AR. Pain in patients with pancreatic cancer: prevalence, mechanisms, management and future developments. Dig Dis Sci 2017; 62(4): 861-870. https://doi.org/10.1007/s10620-0174488-z.

9. Barratt DT, Bandak B, Klepstad P, Dale O, Kaasa S, Christrup LL, et al. Genetic, pathological and physiological determinants of transdermal fentanyl pharmacokinetics in 620 cancer patients of the EPOS study. Pharmacogenet Genomics 2014; 24(4): 185-194. https://doi.org/10.1097/fpc.0000000000000032.

10. Smith MT, Muralidharan A. Pharmacogenetics of pain and analgesia. Clin Genet 2012; 82(4): 321-330. https://doi.org/10.1111/i.13990004.2012.01936.x

11. Sychev DA, Otdelenov VA. Drug interactions in the practice of an internist: a clinical pharmacologist's view. Spravochnik poliklinicheskogo vracha 2014; (12): 18-21. Russian. https://www.elibrary.ru/item.asp?id=23418064.

12. Sychev DA, Sosnovsky EE, Orekhov RE, Bordovsky SP. Contemporary methods of dealing with polypharmacy in elderly and senile patients.
Siberian Medical Review 2016; (2(98)): 13-21. Russian. https://elibrary.ru/item.asp?id=26331298.

13. Farrar JT, Young JP Jr, LaMoreaux L, Werth JL, Poole RM. Clinical importance of changes in chronic pain intensity measured on an 11point numerical pain rating scale. Pain 2001; 94(2): 149-158. https://doi.org/10.1016/s0304-3959(01)00349-9.

14. Azam F, Latif MF, Farooq A, Tirmazy SH, AlShahrani S, Bashir S, et al. Performance status assessment by Using ECOG (Eastern Cooperative Oncology Group) score for cancer patients by oncology healthcare professionals. Case Rep Oncol 2019; 12(3): 728-736. https://doi.org/10.1159/000503095.

15. TNM classification of malignant tumours, 7th ed. Chichester, West Sussex; Hoboken, NJ: Wiley-Blackwell, 2010.

16. Levey AS, Stevens LA, Schmid CH, Zhang YL, Castro AF 3rd, Feldman HI, et al. A new equation to estimate glomerular filtration rate. Ann Intern Med 2009; 150(9): 604-612. https://doi.org/10.7326/0003-4819-1509-200905050-00006.

17. Pancreatic cancer. Clinical guidelines. 2020. Russian. http://cr.rosminzdrav.ru/\#!/recomend/216.

18. Gus'kov SYu, Lyovin VV. Confidence interval estimation for quality factors of binary classifiers - ROC curves, AUC for small samples. Engineering Journal: Science and Innovation 2015; (3(39)): 1-13. Russian. https://doi.org/10.18698/2308-6033-2015-3-1376.

19. Laugsand EA, Skorpen F, Kaasa S, Sabatowski R, Strasser F, Fayers P, et al. Genetic and non-genetic factors associated with constipation in cancer patients receiving opioids. Clin Trans/ Gastroenterol 2015; 6(6): e90. https://doi.org/10.1038/ctg.2015.19.

20. Colombo F, Pintarelli G, Galvan A, Noci S, Corli O, Skorpen F, et al. Identification of genetic polymorphisms modulating nausea and vomiting in two series of opioid-treated cancer patients. Sci Rep 2020; 10(1): 542. https://doi.org/10.1038/s41598-019-57358-y.

21. Corli O, Floriani I, Roberto A, Montanari M, Galli F, Greco MT, et al. Are strong opioids equally effective and safe in the treatment of chronic cancer pain? A multicenter randomized phase IV 'real life' trial on the variability of response to opioids. Ann Oncol 2016; 27(6): 1107-1115. https://doi.org/10.1093/annonc/mdw097.

22. Oosten AW, Oldenmenger $\mathrm{WH}$, Mathijssen $\mathrm{RH}$, van der Rijt CC. A systematic review of prospective studies reporting adverse events of commonly used opioids for cancer-related pain: a call for the use of standardized outcome measures. J Pain 2015; 16(10): 935-946. https://doi.org/10.1016/j.jpain.2015.05.006.

23. Kurita GP, Ekholm O, Kaasa S, Klepstad P, Skorpen F, Sjøgren P. Genetic variation and cognitive dysfunction in opioid-treated patients with cancer. Brain Behav 2016; 6(7): e00471. https://doi.org/10.1002/brb3.471.

\section{Authors:}

Olga P. Bobrova - MD, PhD, Associate Professor, Department of Pharmacology and Pharmaceutical Consulting with a Course in Postgraduate Education, V.F. Voyno-Yasenetsky Krasnoyarsk State Medical University, Krasnoyarsk; Clinical Pharmacologist, Krasnoyarsk Regional Clinical Oncology Center, Krasnoyarsk, Russia. https://orcid.org/0000-00021779-9125.

Sergei K. Zyryanov - MD, DSc, Professor, Chair of the Department of General and Clinical Pharmacology, Peoples' Friendship University of Russia, Moscow; State Clinical Hospital No. 24, Moscow, Russia. https://orcid.org/0000-0002-6348-6867.

Natalya A. Shnayder - MD, DSc, Professor, Leading Researcher of Center of Personalized Psychiatry and Neurology, V.M. Bekhterev National Medical Research Center for Psychiatry and Neurology, Saint-Petersburg; V.F. Voyno-Yasenetsky Krasnoyarsk State Medical University, Krasnoyarsk; Russia. https://orcid.org/0000-0002-2840-837X

Marina M. Petrova - MD, DSc, Professor, Head of the Department of Ambulance Care, V.F. Voyno-Yasenetsky Krasnoyarsk State Medical University, Krasnoyarsk, Russia. https://orcid.org/0000-0002-8493-0058. 